\begin{tabular}{|l|l|l||}
\hline \multicolumn{2}{|c|}{ PublisherInfo } \\
\hline \hline PublisherName & $:$ & BioMed Central \\
\hline \hline PublisherLocation & $:$ & London \\
\hline \hline PublisherImprintName & $:$ & BioMed Central \\
\hline \hline
\end{tabular}

\title{
Neuropeptide treatment inhibits arthritis
}

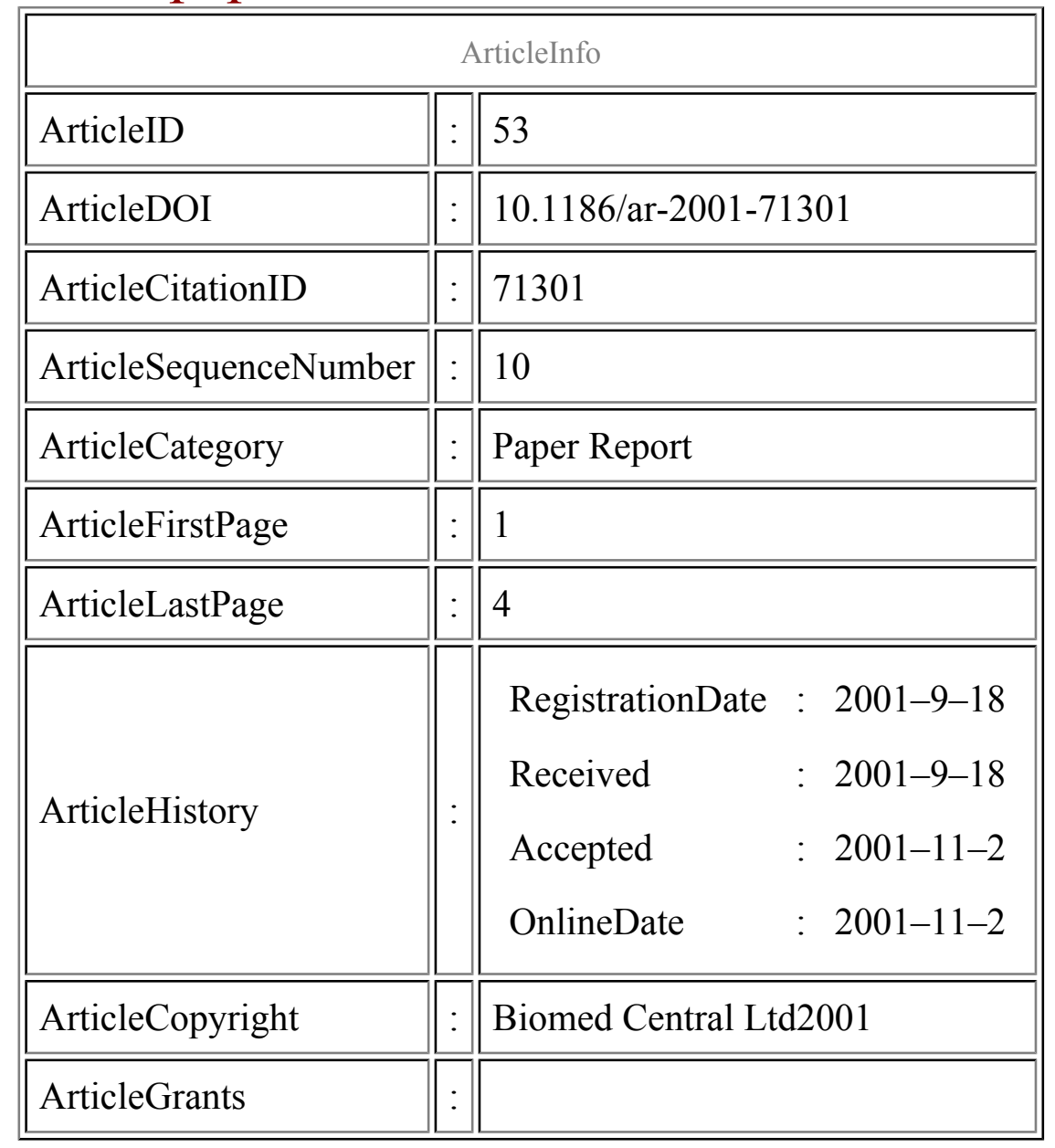




\begin{tabular}{|l|l|l|}
\hline ArticleContext & $:$ & 130753311 \\
\hline
\end{tabular}

Cheryl Smythe, Affl

Aff1 Faculty of Medicine, Imperial College, London, UK

\title{
Keywords
}

\author{
Collagen-induced arthritis, PACAP, Th1/Th2 response
}

\section{Context}

Pituitary adenylate cyclase-activating polypeptide (PACAP) is a 38 amino acid neuropeptide belonging to the secretin-glucagon vasoactive intestinal peptide (VIP) family, which exhibits antiinflammatory properties. PACAP has been shown to protect mice from lethal endotoxemia by decreasing proinflammatory cytokines and increasing anti-inflammatory cytokines. Furthermore, PACAP stimulates a Th2 response. Rheumatoid arthritis (RA) is a chronic inflammatory autoimmune disease in which the imbalance in inflammatory cytokines and T-cell responses play a key role. The authors therefore propose that PACAP may be effective in treating both the inflammatory and autoimmune conditions of RA and investigate this in an animal model of collagen-induced arthritis (CIA).

\section{Significant findings}

The overall incidence and severity of CIA was dramatically reduced in mice treated with PACAP 21 days after primary immunization compared to untreated controls. The joints of treated animals showed an abrogation of synoviocyte proliferation, leukocyte infiltration, pannus formation, cartilage destruction and bone erosion. Administration of PACAP 12 days after disease onset prevented further disease progression with a slight improvement of existing clinical symptoms. PACAP inhibited CIA-induced mRNA expression of the proinflammatory cytokines TNF-a, IL-6, IL-12, iNOS, IL-18, IL-1a and ?, and the chemokines monocyte chemoattractant protein-1, RANTES, MIP-1a and ? and MIP-2. In contrast levels of the anti-inflammatory cytokine IL-10 and the IL-1 receptor antagonist increased following treatment. PACAP inhibited membrane metalloproteinase- 2 mRNA expression and activity. It also affected T-cell responses: T-cell clonal expansion in response to type-II collagen (CII) was partially inhibited; the CIA-induced Th1 response of high IFN-? and low IL-4 was reversed, apparently due to an increase in Th2 IL-4 secreting T cells and a decrease in Th1 IFN-? secreting T cells. Furthermore, anti- 
CII IgG2a levels were significantly reduced, but IgG1 levels increased in PACAP treated CIA mice and there was a decrease in the Th-suppressor ratio as a result of lower levels of $\mathrm{CD}^{+}$cells while $\mathrm{CD} 8^{+} \mathrm{T}$ cell numbers remained unchanged.

\title{
Comments
}

These results demonstrate that administration of PACAP almost completely abrogates disease by altering multiple inflammatory and autoimmune mediators. Similar effects have been previously reported for the related peptide VIP (see Additional infirmation). Significant inhibition of disease does require PACAP treatment prior to disease onset; however, disease progression is prevented if treatment is initiated after disease onset. Remarkably, amelioration of disease persisted 2 weeks after cessation of treatment. Further characterisation of PACAP in alternative models of RA is now required. Whether the incredible efficacy of this agent leaves treated animals immunosuppressed also requires investigation. If treated animals are not immunocompromised and it proves to be well tolerated, this neuropeptide could be a good candidate for human therapeutic development and may well be effective in treating other inflammatory diseases.

\section{Methods}

CIA model, histopathology, RNase protection and Northern analyses, zymography, T-cell proliferation assay, ELISA, synoviocyte culture, flow cytometry

\section{Additional information}

\author{
Delgado M, Abad C, Martinez C, Leceta J, Gomariz RP: Vasoactive intestinal peptide prevents \\ experimental arthritis by downregulating both autoimmune and inflammatory components of the \\ disease.
}

Nat Med 2001, 7:563-568. 


\section{References}

1. Abad C, Martinez C, Leceta J, Gomariz RP, Delgado M: Pituitary adenylate cyclase-activating polypeptide inhibits collagen-induced arthritis: an experimental immunomodulatory therapy. J Immunol. 2001, 167: 3182-3189.

This PDF file was created after publication. 